\title{
Asincronías y desencuentros entre la gestión del riesgo y la participación ciudadana en Chile: apuntes a partir del caso del relleno sanitario Santa Marta $^{1}$
}

\author{
Estela Adasme Calisto ${ }^{2}$ \\ Christian Quinteros Flores ${ }^{3}$
}

\section{RESUMEN}

La Gestión del Riesgo es un concepto ampliamente desarrollado en la esfera privada, que debiera incorporarse progresivamente al ámbito público en la administración de proyectos de impacto ambiental. Ello podría optimizar su alcance al considerar la participación de los reales o potenciales afectados, ya sea a nivel preventivo, evaluativo, o incluso, paliativo. La evidencia empírica indica que en Chile, no solo su aplicación es débil e inconexa entre las distintas instituciones y niveles operativos convocados al momento de generar desarrollo con intervención ambiental, sino que, además, no existen instancias formales y sistemáticas de participación que permitan reducir los riesgos derivados de las amenazas medioambientales. Este artículo pretende mostrar que el involucramiento de los ciudadanos en la gestión de los riesgos es aún muy incipiente y débil, tratándose, más bien, de una visión técnicoacadémica que de una visión política-pública, que aborde integralmente estas situaciones consideradas complejas, desconociendo las potencialidades de este tipo de gestión para el desarrollo.

Palabras Clave: Políticas Públicas, Gestión del Riesgo, Participación Ciudadana

1 Artículo recibido el 26/12/2016. Artículo aprobado el 18/07/2017.

2 Chilena, Trabajadora Social Universidad Santo Tomás, Docente Universidad INACAP, e-mail: eadasmec@yahoo.es

3 Chileno, Trabajador Social Universidad de Valparaíso, Docente Escuela de Gobierno y Gestión Publica EGGP, Universidad de Chile, e-mail: cquinterosflores@gmail.com 


\title{
Assíncronas e desentendimentos entre a gestão do risco e a participação cidadã no Chile: notas a partir do caso do aterro sanitário de Santa Marta
}

\begin{abstract}
RESUMO
A Gestão do Risco é um conceito amplamente desenvolvido na esfera privada, que deve ser progressivamente incorporado ao domínio público na administração de projetos de impacto ambiental. Isso poderia otimizar seu alcance considerando a participação dos reais ou potenciais afetados, seja em nível preventivo, avaliador ou mesmo paliativo. A evidência empírica indica que no Chile, não só a sua aplicação é fraca e desconectada entre as diferentes instituições e os níveis operacionais convocados no momento da geração de desenvolvimento com intervenção ambiental, mas também, não há instâncias formais e sistemáticas de participação que permitam reduzir os perigos produto de ameaças ambientais. Este artigo pretende mostrar que o envolvimento dos cidadãos na gestão de riscos ainda é muito fraca e incipiente, se tratando de uma visão técnico-acadêmica, em vez de uma visão de política pública que aborde integralmente estas situações complexas, ignorando as potencialidades desse tipo de gerenciamento para o desenvolvimento.
\end{abstract}

Palavras-chave: Políticas Públicas - Gestão do Risco - Participação Cidadã

\section{Asynchronies and disagreements between risk management and citizen participation in Chile: Notes from the case of the Santa Marta sanitary landfill}

\section{ABSTRACT}

Risk Management is a widely developed concept in the private field, which should be progressively incorporated into the public domain for the management of projects impacting environment. This could optimize its scope by considering the participation of actual or potential affected people, whether at a preventive, evaluative or even palliative level. The empirical evidence indicates that in Chile, its application is weak and disconnected among different institutions and operational levels convened at the time of generating development with environmental intervention, and, in addition, there are no formal and systematic instances of participation to reduce risks arising from environmental threats. This article aims to show that citizens' involvement in risk management is still very incipient and weak. It is a technical-academic vision rather than a public-policy vision fully addressing these complex situations, ignoring the potential of this type of management for development.

Keywords: Public policies, risk management, citizen participation 


\section{Introducción}

En enero de 2016 se declaró una emergencia de incendio en el Relleno Sanitario Santa Marta, localizado en el sector de Lonquén en la comuna rural de Talagante, Región Metropolitana, uno de los principales rellenos sanitarios construidos en la década anterior. Este depositario de residuos domiciliarios es uno de los más grandes de la región, ya que recibe cerca de 100 mil toneladas de basura de parte de 17 comunas. Inaugurado en el año 2002, nunca ha estado exento de polémica. De hecho, a pocos meses de haberse inaugurado, fue clausurado por no cumplir con todos los requerimientos técnicos necesarios. El incendio -ampliamente informado por la prensa local, regional y nacional- cubrió por cuatro días de gases contaminantes a gran parte de la zona de la Región Metropolitana de Santiago; este hecho visibilizó la peligrosidad de este basural por el impacto de su instalación y los riesgos que representa para la ciudadanía, no solo local sino también, regional. A lo largo de los años, ha habido distintos conflictos en Chile de similar naturaleza, que han involucrado a los vecinos de los territorios afectados. Es así que a partir de estos incidentes surgen algunas interrogantes relacionadas con la metodología utilizada en la política pública para la aprobación y puesta en marcha de los proyectos que representan decisiones de impacto ambiental: ¿Cómo es incorporada la comunidad en estos procesos? ¿Qué tipo de actores comunitarios participan? ¿Solo participa la comunidad aledaña al sector de impacto? ¿Cuáles son las consecuencias de esta gestión? ¿Qué nivel de participación en las decisiones estratégicas tienen la comunidad y sus actores?

\section{¿Cómo se planifica el desarrollo comunal?}

La planificación comunal en Chile depende fundamentalmente de tres instrumentos: el Plan Regulador comunal que asigna y distribuye el uso del suelo, el Plan anual de Inversiones y el Plan de Desarrollo Comunal o PLADECO, este último quizás es uno de los más asociados al desarrollo local y que demuestra mayor grado de participación de la comunidad, aunque como se apreciará en este artícu- 
lo, no está suficientemente vinculado a otras instancias decisionales que afectan el uso de los recursos territoriales en una comuna.

En primer lugar, el ordenamiento territorial de los asentamientos humanos requiere de la tecnificación de la decisión a través de la planificación en sus distintos niveles: local, regional o nacional. Cualquiera sea este, la planificación debe considerar el potencial de desarrollo existente en cada territorio y la capacidad de organización de los actores locales. A nivel de cada localidad, país o territorio, se detecta, por ejemplo, la dotación de una determinada estructura productiva, mercado de trabajo, conocimientos técnicos, capacidad empresarial, recursos naturales, estructura social y política, o tradición y cultura, sobre los que necesariamente articulan las iniciativas locales (Vázquez Barquero, 2007).

Las nuevas estrategias de desarrollo territorial deberán asumir los grandes problemas de organización espacial que se le plantean a una sociedad en la actualidad, tales como las relaciones campo-ciudad, la disyuntiva entre el centralismo y la autonomía regional, la planificación más o menos rígida o ausente, el desarrollo más armónico o crecimiento espontáneo, la descentralización del poder y de las decisiones, las identidades locales y su "defensa" del espacio, todas problemáticas que deben evitar las formas de "fractura territorial" asociadas a la especialización, como señala Arenas (Arenas, 2004).

La revisión bibliográfica permite advertir que si bien la elaboración de estos instrumentos progresivamente involucra a la ciudadanía, a nivel consultivo principalmente, no existe evidencia robusta de que en algunos de los instrumentos de planificación señalados se considere actualmente la participación de los ciudadanos en alguna fase de la gestión del riesgo derivado por amenazas naturales o de deficiente gestión del ordenamiento territorial. Es posible señalar que el riesgo surge de procesos territoriales defectuosos, conductas desordenadas, recursos naturales no controlados, irresponsabilidad social, entre otros factores; por ello es, tanto una fuente de oportunidades como de confrontación, al estar expuesto a los elementos dinámicos de sus procesos. Los últimos 
acontecimientos o catástrofes ocurridas en Chile en los últimos años, indican que las personas se radican en sectores peligrosos o no aptos por distintas razones que develan la insuficiente calidad de las políticas de vivienda o de gestión del riesgo involucrado. Este último aspecto ni siquiera es consultado.

\section{Enfoques para la Comprensión de la Gestión del Riesgo}

La gestión del riesgo es un nuevo problema de planificación que se convierte hoy en una necesidad en toda política pública de desarrollo. Cada vez resultan mayores los espacios donde los vecinos deben ser consultados sobre aspectos propios del desarrollo desde un enfoque territorial. Algunos autores coinciden en afirmar que la gestión del riesgo trabaja sobre situaciones de posible ocurrencia de riesgos y costos no deseados si se dan ciertas condiciones como amenazas y situaciones de vulnerabilidad como pobreza, baja cohesión social o desorganización comunitaria; por tanto, se trata de "Un proceso de adopción de políticas, estrategias y prácticas orientadas a reducir los riesgos asociados a los peligros o minimizar sus efectos" (Cooperación Técnica Alemana, 2004, s/p). También se trata de un proceso en que la sociedad reconoce y valora los riesgos a los que está expuesta y formula, en consecuencia, políticas, estrategias, planes, y realiza intervenciones tendientes a reducir y controlar los riesgos existentes y a evitar nuevos riesgos, "En la medida que busca mejorar las condiciones de vida de la comunidad y proteger tanto la vida misma como el patrimonio individual y colectivo de la población, la gestión del riesgo forma parte de las políticas sociales" (Dirección Desarrollo Urbano y política ambiental, 2005, s/p).

Según Sánchez (2010), el enfoque de análisis del riesgo más común es el denominado naturalista. Este se centra en ofrecer su definición como una "medida", bajo la suposición de que la exactitud debe ofrecerse en la forma de un cálculo. Se concluye que el hombre se encuentra a merced de sufrir riesgos, y por tanto, importa determinar su probabilidad y magnitud (Singh y Ferrer, 
1995; Mc Guirre, 2006; Bryant, 2006, citados en Sánchez, 2010). El desarrollo de los sistemas de información geográfica ha contribuido a que las denominadas "ciencias aplicadas" también hayan seguido y fortalecido este enfoque. De ahí proviene la construcción de mapas de peligro, la elaboración de matrices de daños y curvas de fragilidad.

Una segunda perspectiva de análisis en torno al riesgo, proviene de las ciencias sociales, centrándose en las circunstancias que se conjugan para crear las condiciones necesarias para que los desastres sucedan (Beck, 2006, citado en Sánchez, 2010). Su origen se remonta a la aplicación de la "teoría de la dependencia" a los desastres, entendiendo que éstos son el resultado de procesos económicos y sociales globales, que generarían condiciones de vulnerabilidad de las comunidades frente a eventos naturales. En todo caso, las emergencias naturales o generadas por el ser humano han ido complejizándose e involucrando sistemas no solo antrópicos, sino también tecnológicos. En el cuadro $\mathrm{N}^{\circ} 1$ se aprecia una propuesta de clasificación de los distintos tipos de emergencia que podrían involucrar a la comunidad en su control de daño o en la gestión del riesgo:

Cuadro $\mathrm{N}^{\circ} 1$ Tipos de Emergencia

\begin{tabular}{|l|l|l|}
\hline Tradicionales & Nuevas & Emergentes \\
\hline Inundaciones por cauces & Corte de suministros de & Incendios o explosiones \\
artificiales o lluvias na- & agua potable & de rellenos sanitarios por \\
turales & Caídas de aviones & saturación de residuos \\
Anegamiento por mal uso & Transporte de material & Epidemias \\
de alcantarillado & peligroso en autopistas & Contaminaciones por \\
Temporales de viento & urbanas y vias férreas & uso de pesticidas \\
Incendios de viviendas, & Oleoductos y gasoductos & Emergencias ambienta- \\
pastizales y forestales in- & Densificación en altura & les producto del cambio \\
dustriales & Construcción de mega & climático (aumento de \\
Escapes de gases & proyectos urbanos & marejadas, olas de calor, \\
Accidentes de tránsito & \multicolumn{2}{|l}{ olas de frio) } \\
Guerras & \multicolumn{2}{|l}{} \\
\hline
\end{tabular}

Fuente: Adaptación propia a partir de Sánchez, R. (2010). 
Como resultado, el riesgo se considera en términos de déficit en el desarrollo y no sería más que un conflicto de intereses entre las clases sociales, reflejando la pobreza, marginalidad, mala distribución de los ingresos y exclusión social. Sánchez afirma que "Así es posible señalar que los riesgos y peligros son una construcción social, un proceso que se cimienta por la relación que los hombres establecen con su medio, y que se modifica a través de la evolución sociocultural” (Sánchez, 2010, p. 7). En las últimas décadas, la globalización y el neoliberalismo han exaltado las decisiones individuales en la construcción del territorio, y por lo tanto, se han convertido en causa fundamental del desarrollo del riesgo. Los centros urbanos han adquirido una alta centralización y concentración de funciones y población, de este modo, presentan dos características: en las ciudades se incrementa el riesgo y el peligro, convirtiéndose en un símbolo para la generación de impactos y un lugar privilegiado para la manifestación de desastres, sobre todo los sociales; por otro, son el principal escenario para el desarrollo de oportunidades. La clave en la mitigación de los efectos de un peligro se encontraría en la conformación de asentamientos sustentables y en la construcción de una "cultura de prevención". Sin embargo, es importante reparar en el significado que los sistemas técnico-políticos han asignado a los elementos de emergencia desde la década de 1940, como veremos a continuación. 
Cuadro $N^{\circ} 2$ Progresión de Enfoques

\begin{tabular}{|l|l|l|}
\hline Década & Enfoque & Estrategias \\
\hline 2010 & $\begin{array}{l}\text { Construcción social } \\
\text { del riesgo }\end{array}$ & $\begin{array}{l}\text { Enfoque multidisciplinario del riesgo } \\
\text { Territorialización de las amenazas y vulnera- } \\
\text { bilidades } \\
\text { Integralidad en la gestión } \\
\text { Análisis de capacidades del territorio }\end{array}$ \\
\hline 2000 & Riesgos & Enfoque al desarrollo / Actores del desarrollo \\
\hline 1990 & $\begin{array}{l}\text { Vulnerabilidad } \\
\text { Amenaza }\end{array}$ & $\begin{array}{l}\text { Enfoque social / Académicos de ciencias so- } \\
\text { ciales }\end{array}$ \\
\hline 1980 & $\begin{array}{l}\text { Estudios científicos "exposición", obras inge- } \\
\text { niería } \\
\text { Comités consultivos científicos }\end{array}$ \\
\hline $\begin{array}{l}\text { Preparativos, preven- } \\
\text { ción, mitigación. } \\
\text { Ciclo de desastres, } \\
\text { ayuda humanitaria, } \\
\text { emergencia, recupe- } \\
\text { ración }\end{array}$ & $\begin{array}{l}\text { Organismos ad hoc, Salud, Cruz Roja } \\
\text { Emergencia }\end{array}$ & $\begin{array}{l}\text { Atención a situaciones de guerra, Ejército, } \\
\text { FF.AA. }\end{array}$ \\
\hline 1940 & & \\
\hline
\end{tabular}

Fuente: Elaboración propia a partir de Narváez et al. (2009) y Trejo (2009)

Tal como se aprecia en el cuadro $\mathrm{N}^{\circ} 2$ en materia de enfoques, se ha transitado desde una concepción básica de la emergencia que responsabilizaba a las Fuerzas Armadas y/o militares por el control de la situación de desastre, hacia un enfoque actual más democrático y coparticipativo, como lo son el enfoque de Riesgos y luego el de Construcción Social del Riesgo, donde se aborda la multidimensionalidad del concepto. Este último enfoque involucra un conjunto de actividades que se ha denominado como ciclo del manejo del riesgo y está compuesto por las etapas de Prevención, Respuesta, Recuperación y Reconstrucción.

En este contexto, el desarrollo de capacidades humanas y territoriales constituye hoy una estrategia central para la reducción de riesgos de desastres, lo que hace imprescindible construir habi- 
lidades en las propias comunidades y en las organizaciones, de forma tal, que sean capaces de enfrentar con éxito los riesgos a los que se exponen. La participación ciudadana requiere para su consolidación, formación de personal, la asistencia técnica especializada, el fortalecimiento de las capacidades de las comunidades y de las personas para reconocer y reducir los riesgos de su localidad. En la actualidad se hace imprescindible que se acceda al intercambio informativo, la transferencia tecnológica, el vínculo de actores de la sociedad alrededor de un mismo hecho, como parte del trabajo de instituciones especializadas como apoyo al desarrollo de territorios, que inserte a la gestión del riesgo de manera indisoluble en su quehacer (Gobierno de Costa Rica. Plan Nacional para la Gestión del Riesgo, 2010-2015).

La ciudadanía aporta continuamente experiencias que no deben ser soslayadas, sino incorporadas a las propuestas de reducción de riesgos si son técnicamente viables y garantizan la reducción de vulnerabilidades o la adaptación ante un peligro en particular. Cada propuesta de intervención en el espacio debe llevar consigo la capitalización del acervo histórico cultural de la localidad y empoderarse de él, contribuir a reducir vulnerabilidades, a la vez que pueden ser objeto de análisis e inclusión como "evaluación de riesgos, programas y políticas de intervención”, para su enfrentamiento.

\section{Participación Ciudadana en la Implementación del Relleno Santa Marta}

Si bien debiéramos estar hoy en condiciones de responder a situaciones medioambientales desde un enfoque de "riesgo", aún se sigue abordando este tipo de contingencias desde un enfoque de "Amenaza" propio de los años 80, como se señala en el Cuadro No 2. En efecto, lo ocurrido en la comuna de Talagante así lo indica. La superficie de la comuna alcanza los 125,5 km2 y la mayor extensión comunal corresponde a suelos de alta calidad para la agricultura, que han marcado el carácter productivo de la comuna. La población se concentra fundamentalmente en la ciudad, 
destacándose pocas agrupaciones poblacionales en el resto del territorio, el que se puede caracterizar, más bien, por acoger un proceso de ocupación dispersa, propia de las "parcelas de agrado". El Plan de Desarrollo Comunal de Talagante vigente (2014-2018), uno de los tres instrumentos de gestión municipal establecido en la Ley de Municipalidades (Ley $\mathrm{N}^{\circ} 18.695$ ), contiene las imágenes/objetivo comunales, las sectoriales, la Misión Municipal, los Lineamientos Estratégicos, las Metas y los Indicadores de Verificación. La metodología desarrollada para la obtención de estos productos fue participativa, donde actores comunales, representativos de las diferentes áreas del territorio, plantearon sus perspectivas para cada una de las materias consultadas. Sin embargo, no se aprecia nítidamente la gestión del riesgo en este instrumento ni en un nivel preventivo, de restauración o de rehabilitación de eventuales daños producidos por algunos proyectos de impacto medioambiental. Más bien, sigue una línea normativo-tradicional del desarrollo.

En el documento revisado, se declara la "visión" de Talagante como "Una comuna limpia, con áreas verdes y una infraestructura pública de alta calidad, siempre en la búsqueda del desarrollo sustentable. Altamente participativa, orientada al desarrollo y cercana a las personas" (PLADECO 2014-2018, p. 14).

La metodología de trabajo desarrollada en el instrumento consistió en plantear y agrupar una serie de líneas estratégicas de desarrollo comunal: 
Cuadro Nº 3 Políticas PLADECO TALAGANTE 2014-2018

\begin{tabular}{|c|c|c|c|c|}
\hline $\begin{array}{l}\text { Políticas para } \\
\text { el Desarrollo } \\
\text { Social }\end{array}$ & $\begin{array}{l}\text { Políticas para la } \\
\text { Inclusión }\end{array}$ & $\begin{array}{l}\text { Políticas para el } \\
\text { Ordenamiento } \\
\text { Territorial }\end{array}$ & $\begin{array}{l}\text { Políticas para } \\
\text { el Desarrollo } \\
\text { Económico }\end{array}$ & $\begin{array}{l}\text { Políticas para } \\
\text { el Desarrollo } \\
\text { Institucional }\end{array}$ \\
\hline $\begin{array}{l}\text { Política de } \\
\text { Seguridad } \\
\text { Ciudadana } \\
\text { Política de } \\
\text { Educación } \\
\text { Política de } \\
\text { Salud } \\
\text { Política de } \\
\text { Deportes } \\
\text { Política de } \\
\text { Desarrollo } \\
\text { Social } \\
\text { Política de } \\
\text { Desarrollo } \\
\text { Comunitario } \\
\text { Política de } \\
\text { Cultura }\end{array}$ & $\begin{array}{l}\text { Política de } \\
\text { Personas con } \\
\text { Discapacidad } \\
\text { Política de la } \\
\text { Mujer } \\
\text { Política de } \\
\text { Infancia y } \\
\text { Juventud } \\
\text { Política de } \\
\text { Adulto Mayor }\end{array}$ & $\begin{array}{l}\text { Política de } \\
\text { Desarrollo } \\
\text { Urbano } \\
\text { Política de } \\
\text { Vivienda Social }\end{array}$ & $\begin{array}{l}\text { Política de } \\
\text { Desarrollo } \\
\text { Económico } \\
\text { Política de } \\
\text { Turismo }\end{array}$ & $\begin{array}{l}\text { Política de } \\
\text { Transparencia } \\
\text { Política de } \\
\text { calidad de } \\
\text { atención y } \\
\text { comunicaciones }\end{array}$ \\
\hline
\end{tabular}

Fuente: Elaboración propia a partir de PLADECO Ilustre Municipalidad de Talagante 20142018.

Dentro de la metodología utilizada en la construcción del Plan de Desarrollo se trató, dentro de las "Políticas de Ordenamiento Territorial", el subtema de la planificación urbana -que es el tema que convoca el interés de este artículo-, donde se señala que:

Los aspectos indispensables para sostener el desarrollo comunal son la conexión entre la planificación urbana y las condiciones de la integración social, la vida cultural y el desarrollo de los servicios municipales. Además, la planificación urbana debe responder a las condiciones espaciales y medioambientales, que ayude así a desarrollar, evaluar y ejecutar proyectos urbanos (PLADECO 2014-2018, p. 196).

Sin embargo el problema central identificado en el área del desarrollo urbano se refirió básicamente al "Déficit de Infraestructura Urbana de Calidad" y a las "Políticas gubernamentales donde la municipalidad no tiene mayor incidencia”, lo que deriva según 
los consultados en uno de los principales problemas asociados a la gestión de recursos en esta área, además de un "Alto nivel de centralidad en las decisiones para el Desarrollo Urbano", teniendo como efecto la "Falta de autonomía municipal para incidir en proyectos de gran impacto comunal (por ejemplo, autopistas)" (PLADECO 2014-2018, p. 197).

Dentro de los objetivos estratégicos de este plan se observa el querer:

Mejorar la calidad de vida de los habitantes de Talagante, a través del desarrollo urbano sustentable y de la promoción de oportunidades de regeneración urbana, y de la aplicación de estrategias de desarrollo local que incorporen criterios de integración funcional, mixtura social, diseño urbano y habitacional, considerando la participación de la comunidad, además de promover y generar en la comuna condiciones para el aumento de inversiones e instalación de servicios y equipamientos que ayuden a mejorar la calidad de vida de los habitantes (PLADECO 2014-2018, p. 197).

También es parte de los objetivos específicos de esta área en el Plan, la promoción de participación y generación de relaciones vecinales entre las diferentes zonas de la comuna, consolidando barrios con diferentes estilos, reconociendo su historia comunitaria, valores, prácticas, tradiciones, respetando y escuchando las opiniones y decisiones de la comunidad" (PLADECO 2014-2018, p. 197).

Por su parte, los lineamientos estratégicos, en general, gravitan en torno a tres tipos de estrategias que pueden ser resumidas en la forma siguiente: Movilización, educación y empoderamiento de la comunidad en materias de Desarrollo Urbano, estrategias de mejora en Gestión del Desarrollo Urbano entre el municipio y la comunidad y la organización de los recursos humanos y materiales del municipio en pos del cumplimiento de metas de gestión correspondientes a la Gestión del Desarrollo Urbano (PLADECO 2014-2018, p. 206). Es decir, la preocupación central de este instrumento en materia de ordenamiento territorial, está referida básicamente a la gestión del desarrollo urbano y no rural del territorio, que es preci- 
samente donde la evidencia arroja que existe la mayor cantidad de problemas de amenazas y vulnerabilidades en materia de riesgo. $\mathrm{Si}$ bien se señalaba en apartado anterior de este artículo que la población de la comuna se concentra fundamentalmente en la ciudad o espacio urbano, esto no debiera descuidar la gestión de localidades más apartadas cuya intervención impacta de igual manera a las zonas urbanas a través de napas subterráneas, ruidos, malos olores o emergencias de incendios de tipo industrial como es nuestro caso de análisis. La sustentabilidad medioambiental debe preocuparse de la capacidad del territorio en forma integral, lo que significa que debe articular adecuadamente la mayor cantidad de instrumentos de planificación disponibles. Por otra parte, en la revisión del instrumento no se aprecia preocupación por incorporar sistemáticamente en algún proyecto a la comunidad local en la gestión de los proyectos de impacto ambiental en la comuna.

Respecto de los procesos de gestión participativa, el PLADECO comunal señala que la participación en este instrumento se orientó a formar la visión comunal de Talagante:

Que representa los sueños y anhelos que poseen los actores que dan vida a la comuna de Talagante (comunidad, funcionarios municipales, alcalde, concejales, entre otros), esta visión se ha construido a través de la realización de una serie de trabajos participativos, aplicando distintas metodologías, entre las que se cuentan entrevistas, grupos focales, encuesta, cabildos territoriales y talleres con funcionarios municipales (PLADECO 2014-2018, p. 10).

Tampoco se aprecia a lo largo del documento la existencia de metodologías participativas o instancias de acción vecinal permanentes que monitoreen el desempeño, por ejemplo, de la operación del citado relleno sanitario y de todas estas problemáticas señaladas.

Por otra parte, y en relación al otro instrumento de planificación comunal, no se aprecia vinculación entre el PLADECO aludido y el Plan Regulador Comunal, el segundo instrumento contemplado en la Ley de Municipalidades (Ley 18.695) y cuya elaboración 
y gestión es una función privativa del municipio en Chile, evidenciando un absoluto desencuentro entre los instrumentos de gestión local del territorio y sus habitantes.

Sin embargo, y tal como se señalaba al inicio del artículo, se evidencia una ausencia de la incorporación del concepto de gestión de riesgo, tanto dentro del marco del ordenamiento territorial comunal, como del citado PLADECO. La evidencia indicaría que, al parecer, sólo se considera a la comunidad cuando ocurre una tragedia (nivel de respuesta) como lo acontecido en el incendio del relleno Santa Marta, ya que a pesar de que la comunidad participó en el proceso de evaluación de impacto ambiental, solo se la involucró desde una lógica más bien informativa y de consulta, pero sin poder decisional asociado a una participación permanente y gestionaria. La comunidad expresada en comités de agua potable rural, asambleas ciudadanas, juntas de vecinos, no es consultada en la definición y localización de estos centros de residuos, como tampoco el gobierno local de Talagante, aun cuando se puede apreciar que sus políticas de residuos domiciliarios a nivel vecinal manifiestan un avance en este tipo de gestiones en cuanto a fortalecer la responsabilidad social por parte de los vecinos en el cuidado del medioambiente (PLADECO 2010-2014). Tampoco se involucra a actores de otras comunas en estas fases.

En materia de aprobación de proyectos específicos asociados al uso del territorio, se constata que en el proyecto Santa Marta tampoco hubo importantes niveles de participación ciudadana. Por ejemplo, todo proyecto de impacto ambiental supone el siguiente ciclo de vida útil que comienza con la aprobación del proyecto por parte de la autoridad, luego, por la etapa de construcción, seguida por la de implementación, para finalmente llegar a la etapa de abandono. (Ver figura $\mathrm{N}^{\circ} 2$ ). 
Figura $N^{\circ}$ 4: Ciclo del Proyecto Relleno Sanitario Santa Marta

Ciclo del Proyecto Relleno Sanitario Santa Marta

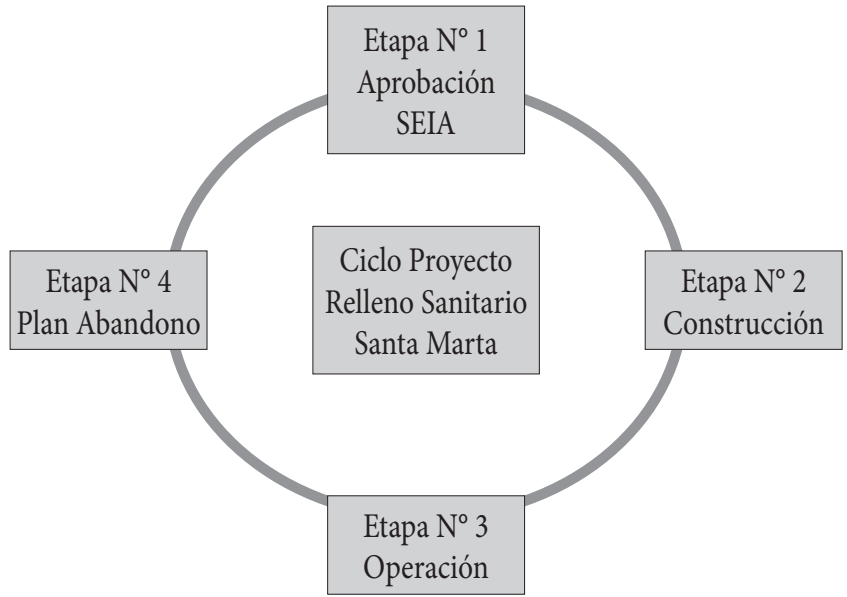

Fuente: Elaboración propia en base al Informe Técnico del Estudio de Impacto Ambiental (EIA) del Proyecto "Relleno Sanitario Santa Marta" 2001. COREMA Metropolitano.

En este caso y dado el análisis de la evidencia empírica y documental revisada por los autores del presente artículo, se puede concluir que el proceso de consulta inicial fue bastante débil, remitiendo básicamente a la implementación de un sistema de correspondencia abierto con los responsables del proyecto pero que en las fases posteriores tampoco se consolidó como se advierte en la figura $\mathrm{N}^{\circ} 3$ : 
Figura $\mathrm{N}^{\circ}$ 5: Participación de la Comunidad en el Ciclo del Proyecto

$$
\text { Evaluación Impacto Ambiental }
$$

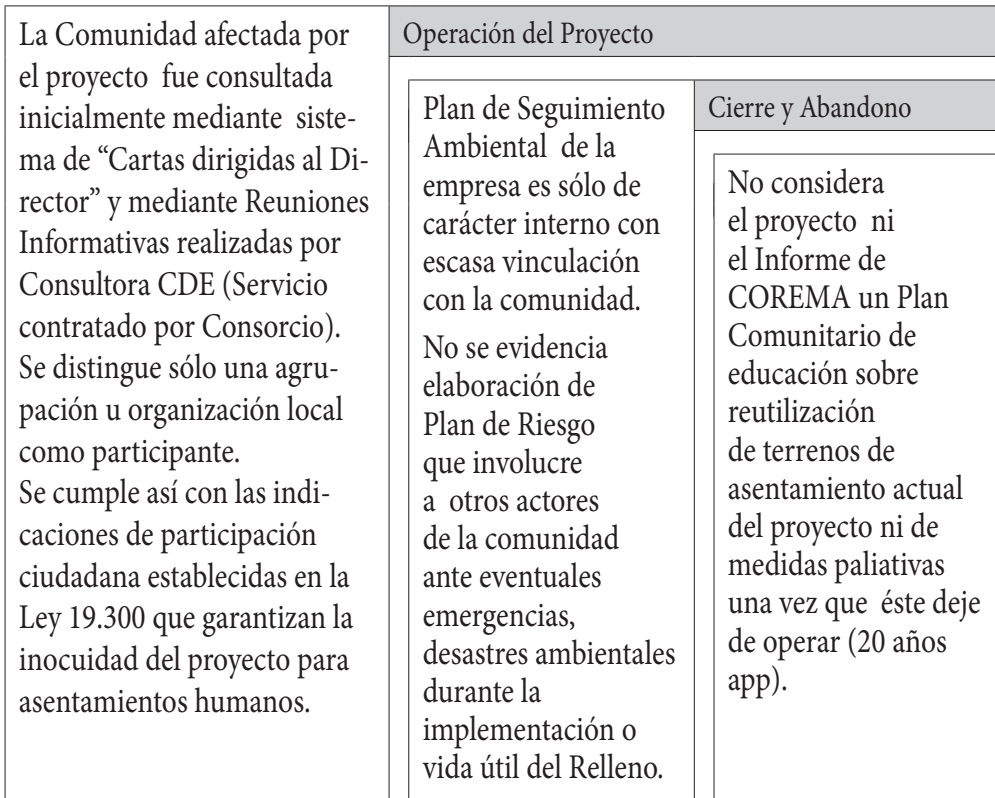

Fuente: Elaboración propia en base al Informe Técnico del Estudio de Impacto Ambiental (EIA) del Proyecto "Relleno Sanitario Santa Marta" 2001. COREMA Metropolitano

Específicamente, en cuanto al proyecto del relleno Santa Marta, es posible observar (Figura $\mathrm{N}^{\circ} 3$ ) una participación parcial de la comunidad en todo el ciclo de vida del proyecto. Desde que inició sus operaciones en 2002, la incorporación de la comunidad se ubicó sólo en la etapa de diseño del proyecto en el marco de la evaluación de impacto ambiental, pero luego de la aprobación técnica del proyecto por parte de la autoridad competente, Servicio Evaluación Ambiental (Ley 20.417), no existió un plan para incorporarla en las etapas de operación o en un plan de vinculación comunitaria en caso de abandono o cierre del proyecto. La vinculación que actualmente sostiene la empresa Consorcio Santa Marta con la comunidad obedece más bien al concepto de RSE (Responsabilidad Social Empresarial) más que a una voluntad de incorporar a los ciudadanos en un plan de riesgo integral y participativo. 


\section{Niveles en la Gestión del Riesgo en Chile}

La potencial inclusión de la gestión del riesgo en Chile encuentra, primero, un problema central que es la falta de conexión entre los distintos actores que participan en ella. Municipalidades con instrumentos limitados, superintendencias que autorizan proyectos sin consulta local, excesiva centralización administrativa de las decisiones, entre otros. La legislación chilena contempla diferentes normas sobre la fiscalización y coordinación entre distintas autoridades públicas al momento de gestionar situaciones de riesgo producto de condiciones medioambientales o de precaria organización territorial. Al respecto, hay que tener presente la existencia de la Superintendencia de Medio Ambiente, creada por la Ley $\mathrm{N}^{\circ}$ 20.417, a la cual corresponde fiscalizar las Resoluciones de Calificación Ambiental, de las medidas de los Planes de Prevención y/o de Descontaminación Ambiental, del contenido de las Normas de Calidad Ambiental y Normas de Emisión, y de los Planes de Manejo, cuando corresponda, y de todos aquellos otros instrumentos de carácter ambiental que establezca la ley.

Por otra parte, según el Código de salud, en particular el artículo 36, corresponde a la Seremi de Salud declarar zona de emergencia sanitaria, sobre todo cuando producto de un incendio, por ejemplo, la población podría ser afectada. Ahora, la Intendencia Regional (un nivel regional del sistema presidencial), en virtud de lo dispuesto en el artículo 2 de la ley Orgánica Constitucional sobre Gobierno y Administración Regional, podrá ejercer la coordinación, fiscalización o supervigilancia de los servicios públicos creados por ley para el cumplimiento de la función administrativa que operen en la región, como también, adoptar todas las medidas necesarias para prevenir y enfrentar situaciones de emergencia o catástrofe.

En lo que respecta a los municipios locales, la Ley Orgánica Constitucional de Municipalidades, señala en el artículo 4, que las municipalidades podrán desarrollar con otros órganos de la administración del Estado, funciones relacionadas con la prestación de 
auxilio en situación de emergencia, como también, en la protección del medio ambiente.

Ahora bien, en lo que concierne a la participación ciudadana en la gestión pública (sin considerarla aún en la gestión del riesgo), se constata la importancia que le han concedido los últimos gobiernos, toda vez que las relaciones del Estado y la ciudadanía se desarrollan en una nueva situación que involucra lo social y lo político, desde una visión que permita el acceso, reconocimiento y ejecución de los derechos ciudadanos. Mecanismos como Oficinas de Información, Reclamos y Sugerencias, OIRS, Audiencias, Consejos de la Sociedad Civil, entre otros, evidencian esta nueva realidad.

Por ello, se debe considerar la participación ciudadana como un elemento central de la formulación de políticas, programas y proyectos, apuntando a instalar mecanismos formales de interlocución entre los distintos niveles del Estado y los ciudadanos con la finalidad de que puedan influir en las distintas fases de toma de decisiones (diseño, ejecución y seguimiento), aumentando los niveles de control social sobre las políticas públicas, donde lejos de debilitar al Estado y su funcionamiento, contribuyen a la legitimización del mismo, aumentando la eficacia de su desempeño.

$\mathrm{Al}$ respecto, las iniciativas, orientaciones y políticas en materia ambiental no han estado ajenas a esta tendencia de diseño de planes públicos, lo cual es posible observar en la Ley 19.300 (párrafo $3^{\circ}$, de la participación de la comunidad en el procedimiento de evaluación de impacto ambiental), donde se explicita el procedimiento que deben desarrollar los proyectos que tienen impacto ambiental a nivel comunitario, reforzado por la ley 20.417, donde modifica varios de sus artículos para potenciar el rol de la ciudadanía en el proceso de evaluación de impacto ambiental, como también, despolitizar la Evaluación de Impacto Ambiental (EIA) y aumentar la eficiencia en los procedimientos. Para ello se crea el Servicio de Evaluación Ambiental con énfasis técnico (SEA), que racionaliza y centraliza la fiscalización ambiental, anclado en la Superintendencia del Medio Ambiente (SMA), e instala la Evaluación Ambiental Estratégica (EAE). 
En materia de salud pública es interesante considerar lo planteado por la Organización Mundial de la Salud (OMS) respecto de que las amenazas colectivas al bienestar de las comunidades, se pueden considerar como formas de violencia. Efectivamente y según el Informe mundial sobre la violencia y la Salud de la OMS, la violencia se puede entender como:

El uso deliberado de la fuerza física o el poder, ya sea en grado de amenaza o efectivo, contra uno mismo, otra persona o un grupo o comunidad, que cause o tenga muchas probabilidades de causar lesiones, muerte, daños psicológicos, trastornos del desarrollo o privaciones (OMS, 2002, p. 15).

Asimismo, señala la OMS, que la definición de violencia "involucra a toda deficiencia que se produzca en el marco del desarrollo "y que comprometa el bienestar de los individuos, las familias y las comunidades" (OMS, 2002, p. 17). Según la propia OMS, la violencia es un problema polifacético, para el que no existe una solución sencilla o única; antes bien, como pone de manifiesto el modelo ecológico empleado en el informe, es necesario actuar simultáneamente en varios niveles y en múltiples sectores de la sociedad para prevenirla, tanto desde factores de riesgo individuales, como comunitarios.

\section{Mecanismos de Participación Ciudadana para la Gestión Local del Territorio}

No obstante todo lo anterior, y tal como se ha señalado, el uso del territorio comunal está definido en gran parte en Chile por los planes reguladores comunales y los Planes de Desarrollo Local. Sin embargo, la calidad de estos instrumentos depende muchas veces de la discrecionalidad del alcalde de turno y de su convicción personal sobre la participación ciudadana. La actual política de desarrollo urbano declara que:

Chile no es un país acostumbrado a la dictación de "políticas" en este ámbito, en cuanto a iniciativas que tengan las condiciones y el sustento necesario para su materialización. Una 
política no es una ley o un reglamento sino un documento dirigido al país en el cual se establecen principios, objetivos y líneas de acción con el fin de ayudar a mejorar la calidad de vida de todos. Por lo mismo su fuerza o debilidad, su permanencia o transitoriedad, dependen del valor que a dichos principios y contenidos otorguen los propios chilenos (Política Nacional de Desarrollo Urbano, 2014, p. 13).

En cuanto a desarrollo urbano la formulación de una política es especialmente compleja, toda vez que históricamente las políticas en esta materia han estado centradas más bien en aspectos urbanísticos, de zonificación y de calidad de la construcción. Una política de desarrollo urbano local debiera hoy centrarse en la persona, considerar las creencias de las comunidades, y los factores que afectan la calidad de vida de los habitantes. Es así que el ordenamiento territorial en Chile, se debería iniciar desde el propio espacio local a través de la aplicación del Plan Regulador Comunal, en base al cual, la autoridad debiera definir participativamente el uso del suelo, las áreas de expansión, el acceso a los servicios, las zonas de utilidad pública, entre otros; impactando directamente en la calidad de vida de los habitantes. Las posibilidades de que la comunidad participe actualmente en la elaboración o modificación de estos instrumentos de planificación urbana, a veces se refieren solo a votaciones populares respecto de la pertinencia del cambio en el uso de suelo, pero sin mayor trascendencia e impacto en la decisión final que siempre resulta técnica, política o económica. Por otra parte, las autoridades locales no son capaces de "prevenir" la ocurrencia de ciertas desavenencias entre vecinos producto de este ordenamiento o ineficiente distribución del espacio.

La gestión eficiente del riesgo presenta una directa relación con los nuevos desafíos de la convivencia contemporánea, no solo haciendo técnicamente más útil el uso del suelo y sus recursos sino también respondiendo a las nuevas demandas de los ciudadanos de intervenir e influir en las decisiones que los impactan. Una adecuada calidad de vida implica el logro de procesos de inclusión e integración, con distancia de la fragmentación o exclusión social. 
Una de las preocupaciones de la modernización es cómo generar desarrollo desde el punto de vista objetivo y subjetivo. La distribución y uso del espacio es un indicador del componente subjetivo de la calidad de vida al generar, en el ciudadano, un sentido de pertenencia y de afiliación a sus comunidades, a sus costumbres, permitiendo el ejercicio de una ciudadanía plena, respetuosa del entorno y de los recursos naturales.

De esta forma, las estrategias de desarrollo local conviene plantearlas de forma diferenciada, reconociendo las necesidades y demandas particulares de las localidades y territorios. La ciudad solo puede ser el espacio de libertad individual, de cohesión social y un lugar de progreso económico-social si existen las condiciones mínimas de participación cívica; "la ciudad es una construcción política”, como señala Aristóteles. Tal como indican Smolka, M.O. \& Mullahy (2007), esta nueva situación reaviva con fuerza y justifica el renovado interés por el manejo del suelo como pieza estratégica dentro del abanico de las políticas públicas territoriales.

\section{Reflexiones finales}

Sin duda que lo ocurrido en el proceso de implementación del Relleno Santa Marta de Lonquén, es un caso emblemático para evidenciar la existencia de una débil institucionalidad para la participación ciudadana en la gestión del riesgo, en este caso en particular, en la implementación de un relleno sanitario, pero que perfectamente pudiera ampliarse a otras situaciones donde el riesgo se debe administrar cada vez más con participación de los distintos actores que intervienen en el desarrollo. Tal es el caso de situaciones de construcción de viviendas y equipamiento comunitario en cursos de ríos secos, o en el piedemonte de una localidad, o en quebradas de cerros como ha sido el lamentable caso de lo ocurrido recientemente en Valparaíso en materia de incendios forestales con impacto residencial. Se trata, pues, de una situación que podría replicarse en otros contextos similares en el país. La institucionalidad chilena, en este caso, privilegiaría la participación comunitaria en la fase inicial de todo proyecto con impacto ambiental. Así lo manifiestan los SEIA 
(Sistemas de Evaluación de Impacto Ambiental) que incorporan a los actores de la comunidad en la fase de diagnóstico.

Sin embargo, las reglas del juego actuales no obligan a las empresas responsables de implementar una política pública, a convocar en otras oportunidades a los vecinos del sector o afectados, en un proceso de monitoreo ciudadano o de educación respecto del cierre o abandono de un proyecto. En consecuencia, el análisis de este caso nos plantea que falta incorporar a las comunidades locales en planes que apunten a la visibilización de los riesgos medioambientales, a su cuidado, y no solo como refractarios ante una emergencia sanitaria o de otro tipo.

El conflicto entre actores involucrados en el relleno Santa Marta ha devenido en un conflicto de intereses entre grupos económicos y los vecinos defensores de su territorio. La participación de la comunidad en este tipo de proyectos se realiza en el marco de una institucionalidad en cierta forma inconexa: por una parte el municipio local no tiene ninguna injerencia en la aprobación de proyectos medioambientales con la envergadura de Santa Marta, que, básicamente se rige por disposiciones de la Ley de medioambiente y es fiscalizado por la Superintendencia solo desde un punto de vista técnico. Aunque la comunidad participe en la gestión municipal no podrá influir en las decisiones de instalación de este megaproyecto pues presentan regulaciones distintas que carecen de convergencia.

Si bien se han abordado los dos instrumentos de gestión municipal que claramente no han incorporado la gestión del riesgo, en el caso de Talagante, nada hace mirar con optimismo que esto sí se vaya a aplicar en otras comunas.

La comunidad debe desarrollar su capital sinergético como lo plantea Boisier (1999) e involucrarse como un actor clave del desarrollo Territorial y de esta forma, cuestionar lo planteado por la autoridad local en el PLADECO comunal de Talagante, donde se afirmaba en 2010 que "Existe una carencia de participación efectiva de la comunidad en el desarrollo comunal” (PLADECO 2010-2014). 
En consecuencia, es razonable plantear que debe generarse un plan de gestión del riesgo desde el gobierno local que incluya a la sociedad civil y a sus actores, tanto en las decisiones asociadas a la localización, como en la ejecución o control de daños, la evaluación del impacto socio-ambiental de los distintos proyectos de impacto como rellenos sanitarios, plantas energéticas, megaproyectos, considerando que cada vez más los espacios rurales son absorbidos por los urbanos. Tales restos no solo están relacionados con las intervenciones locales sino que, además, demandan nuevos desafíos en el campo del diseño de políticas públicas, específicamente medioambientales, ya que a pesar de los cambios incorporados a la ley 19.300, aún están ausentes los procedimientos de control y seguimiento de los planes de mitigación de los proyectos desde la comunidad en el ejercicio esperable de su derecho ciudadano. No basta solo con convocar a la comunidad a espacios de participación informativos en la etapa de evaluación de impacto ambiental, los proyectos deberían diseñar estrategias de vinculación con la comunidad de carácter permanente, tanto en su diseño y operación, como ante un eventual cierre o abandono de operaciones del proyecto, ya que son los propios ciudadanos quienes se mantienen en este hábitat.

Pareciera ser, a partir de lo tratado en este artículo, que finalmente solo se consulta a la comunidad en la gestión de la "emergencia ambiental", pero fuera de una concepción integral y sustentable de la gestión del riesgo, instancia previa y permanente que debe instalarse como política medioambiental con un alto valor cultural en la gestión pública, en sus distintos niveles operativos, minimizando progresivamente estas asincronías y desencuentros entre la gestión del riesgo y la participación ciudadana en Chile. La evidencia empírica indica que la gestión del riesgo no sólo está bastante debilitada e inconexa entre las distintas instituciones y niveles operativos convocados al momento de generar desarrollo con intervención ambiental, sino que, además, no cuenta con instancias formales y sistemáticas de participación que permitan reducir los riesgos derivados de las amenazas medioambientales. 


\section{Bibliografía}

Arenas, F. et al. (2004). El Ordenamiento Territorial: Un nuevo tema para la planificación. Ed. Universidad Católica. Págs. 201-214. Santiago de Chile.

Bellet Sanfeliu, C. et al. (2002). Las nuevas funciones urbanas: gestión para la ciudad sostenible, Las líneas de trabajo del programa UIA-CIMES: Ciudades intermedias y urbanización mundial: CEPAL - SERIE Medio ambiente y desarrollo $\mathrm{N}^{\circ} 48$.

Boisier, S. (1999). El Desarrollo Territorial a partir de la Construcción de Capital Sinergético. Estudios Sociales $\mathrm{N}^{\circ}$ 99, Santiago de Chile. COREMA Metropolitano (2001).

Informe Técnico del Estudio de Impacto Ambiental (EIA) del Proyecto "Relleno Sanitario Santa Marta". Consorcio Santa Marta S.A. 30 de julio de 2001.

Cooperación Técnica Alemana - GTZ (2004). Conceptos asociados a la gestión del riesgo de desastre en la planificación e inversión del desarrollo. Dirección General de Programación Multianual del Ministerio de Economía y Finanzas. Programa Desarrollo Rural Sostenible.

Dirección Desarrollo Urbano y Política Ambiental (2005). Guía para orientar las acciones e inversiones en Gestión Local del Riesgo a nivel municipal, Colombia.

Gobierno Costa Rica (2010). Plan Nacional para la Gestión del Riesgo. Marco estratégico para la aplicación de la política de Gestión del Riesgo, 2010-2015.

Ilustre Municipalidad de Talagante (2015). Políticas para el desarrollo 2014-2018, CGA Ingenieros Consultores. Chile. Ley $\mathrm{N}^{\circ}$ 19.300 Sobre Bases Generales del Medio Ambiente. Santiago de Chile.

Ley N¹8.695 Orgánica Constitucional de Municipalidades. Santiago de Chile.

Ley No 19.418 Sobre Junta de Vecinos y demás Organizaciones Comunitarias. Santiago de Chile.

Ley N 19.175 Orgánica Constitucional sobre Gobierno y Administración Regional. Santiago de Chile.

Narváez, L. et al. (2009). La Gestión del Riesgo de Desastres: Un enfoque basado en procesos. Secretaría General de la Comunidad Andina, Perú.

Organización Mundial de la Salud (2002). Informe Mundial sobre la violencia y la salud: Sinopsis. World Report on Violence and Health: Summary ISBN 9241545623. 
Plan de Desarrollo Urbano o la Propuesta de Uso de Suelo para la Integración social (2015). Informe Final. Consejo Nacional de Desarrollo Urbano. Santiago de Chile.

PNUD (2012). Informe sobre Desarrollo Humano en Chile. PNUD. Santiago de Chile.

Rodríguez Otero, C. (2009). Documento de Trabajo Módulo Nº 3. Maestría en Dirección y Gestión Pública Local. La Antigua, Guatemala, 24 al 26 de agosto del 2009.

Rebolledo, J. (2015). Documento de Trabajo Curso Gestión del territorio con Sostenibilidad. Doctorado en Ordenamiento Territorial y Desarrollo Sostenible. Universidad Nacional de $\mathrm{Cu}$ yo.

Sánchez, R. (2010). La debilidad de la gestión del riesgo en los centros urbanos. El caso del Área Metropolitana de Santiago de Chile, en Revista de Geografía Norte Grande. $\mathrm{N}^{\circ}$ 47. pp. 5-26. Departamento de Geografía, Universidad de Chile (Chile).

Smolka, M.O. \& Mullahy, L. (2007). Perspectivas urbanas: temas criticos en políticas de suelo en América Latina / editado por Lincoln Institute of Land Policy. Consultado el día 22 de octubre de 2015 en https://www.lincolninst.edu/pubs/ dl/1180_639_Chapter\%205.pdf

Trejo, R. (2009). La Reducción del Riesgo a Desastres Naturales en las Américas reduce los movimientos migratorios y su impacto ambiental negativo. Departamento de Desarrollo sostenible, extraído el 05 de agosto de 2017 en scm.oas.org/ pdfs/2009/CP21540T.ppt

Vázquez-Barquero, A. (2009). Desarrollo Local: Una Estrategia para Tiempo de Crisis, Universitas Forum. 